of the A-strain fibrosarcoma with C. parrum.

These simple experiments illustrate that tumours growing in their host of origin do not necessarily engender that immunological antipathy in the host lymphoid system which can easily be demonstrated against malignant foreign grafts. Furthermore, the adjuvant bacterium, $C$. parium, need not necessarily be thought of as a T-cell stimulant.

\section{CELL BIOLOGY}

\section{Tubules and Filaments}

from a Correspondent

Blologists with a wide range of backgrounds met to discuss the structure and function of microtubules and microfilaments at the York meeting of the British Society for Cell Biology on September 20 and 21. A. Forer (York University, Ontario) opened the meeting by presenting evidence that actin-like microfiliments interact with microtubules and speculated that these two components act like 'muscle' and 'bone' respectively. He drew attention to the clear zone around the outside of microtubules, and this feature was also present in the electron micrographs of several other speakers. Very little seems to be known about the structure and function of this zone, but it seems to be of sufficiently regular appearance to merit more study.

At the biophysical level, there seemed to be reasonable agreement between several speakers (D. Chasey, University of Edinburgh, E. O'Brien, King's College, London, L. Amos and R. Linck, MRC Laboratory of Molecular Biology, Cambridge) that, at least in the case of some ciliary microtubules, the structure is built up from a 3 start, 13 fold. left handed helix and it seems from preliminary evidence of O'Brien that in vitro reconstructed microtubules may have a similar structure. The study of the ultrastructure of microfilaments is technically even more difficult than that of microtubules, but D. Gilbert (King's College, London) demonstrated that neurofilaments from a marine worm are protein $\alpha$ helices supercoiled into several higher order helical forms. Research on microfilaments is impeded because of the lack of a specific disruptive agent equivalent to colchicine in the study of microtubules. Early optimism of some workers that cytochalasins may have specific effects on microfilaments seems to be fading, to judge by the discussion after a contribution from J. C. Appleton and R. B. Kemp (University College of Wales, Aberystwyth) showing effects of cytochalasin A on cell aggregation.

M. Jacobs (King's College, London) described a careful study of the exchange reactions between the two nucleotide binding sites on the tubulin dimer; the significance of transphosphorylation between the sites during microtubule assembly does not seem clear. W. W. Franke and J. Stadler (University of Freiburg) have extended their work on colchicine-binding properties of membrane fractions and find that such binding may be distinguished from that of tubulin on several grounds, including binding of lumicolchicine.

Several workers approached the problem of interaction between microtubules. J. Cachon (Villefranche-surmer, paper read by $M$. Sleigh) provided a possible explanation of complex microtubule patterns in the axopods of radiolarians in terms of different tubules being 'divalent' or 'trivalent' in their binding properties for other microtubules. J. Tucker (University of St Andrews) extended this discussion by speculating on the roles of templates and nucleating sites, as well as self assembly links, in the formation of microtubule arrays. Several factors can be involved in one organelle. A. C. Crossley (University of Sydney) presented evidence that microtubules can interact over considerable distances (around $800 \AA$ ) during myoblast development.

The potential value and difficulties of $g \in$ netic approaches to microtubule formation were reviewed by J. R. Warr (University of York), who argued that mutants resistant to anti-mitotic agents may provide useful tools for such studies. In contrast with the situation in mammalian cell cultures, colchicine resistant mutants in Chlamydomonas seem not to be simply mutants with permeability defects and consequently may have microtubule defects.

Two botanists, A. W. Robards (Uni- versity of York) and L. Goosen-de-Roo (Leiden), were in broad agreement with Forer who earlier suggested a structural rather than a contractile role for microtubules. Goosen-de-Roo presented a careful series of electron micrographs which support the proposition that rows of microtubules act during thickening of tracheary elements by forming barriers to guide vesicles to required locations.

\section{PALAEOBOTANY \\ Florn of Siberia}

from our Soviet Correspondent THE first detailed results have been published (Dokl. Akad. Nauk SSSR, 209, 1464; 1973) of the pollen and spore content of the stomach of the frozen remains of a horse found in a mine of the Selerikan basin in Yakutia (Siberia) in December 1968. The remains were dated to an absolute age of 37,000 years by the Laboratory of Absolute Geochronology at Leningrad University, a date confirmed by a control determination by Professor 1. Harrington of the National Museum, Ottawa.

The pollen and spore analyses were carried out at the Komarov Botanical Institute of the Academy of Sciences of the USSR. Although further, more detailed, pollen analysis is still in progress, results to date show that the Selerikan horse may prove as significant for an understanding of the palaeobotany of Siberia as did the Beresovka mammoth. Like the Beresovka find, the Selerikan horse reveals an ecological picture typical of present-day Siberia. So far, seventy-two different types of

\title{
X-ray Structure of Antibody
}

THE first X-ray structure determination of the $\mathrm{Fab}^{\prime}$ portion of an immunoglobulin of known antigen specificity has been accomplished by Padlan, Segal, Spande, Davies, Rudikoff and Potter at a resolution of $4.5 \AA$ and appears in Nature New Biology next Wednesday (October 10).

As with chymotrypsin, lysozyme, and so many other proteins, it transpires once more that the molecule is made up of two globular halves of roughly the same size, each of them in this case being in turn subdivided into two domains. This of course accounts well for the four structural elements known to make up the Fab' fragment, namely the variable and constant parts of the light chain, and the corresponding variable and constant parts of that portion of the heavy chain (Fd') with which the light chain makes contact. These four parts can be regarded as centred on the vertices of an elongated tetrahedron, with dimensions of $40 \times 50 \times 80 \AA$.

The other interesting result, which emerges from difference Fourier maps, is the location of the binding site of phosphorylcholine, the specific hapten of this myeloma immunoglobulin. Competition experiments have allowed the authors to distinguish between a nonspecific site, located on the surface of the molecule in the constant region, and the true antigenic binding site. Again, as repeatedly found in enzymes, the striking feature of the specificity site is its location at the bottom of the deep cleft which divides the molecule into its domains. In the case of the immuno. globulin the site is sandwiched by the two masses making up the variable region and formed by elements of both light and heavy chains.

The absence of any important features in the difference map, other than the peaks corresponding to the ligand itself, is taken to indicate that the attachment of the hapten is not accompanied by any large conformational readjustments, such as occurs in allosteric species as, for example, haemoglobin. 